Check for updates

Cite this: RSC Adv., 2018, 8, 27191

Received 1st July 2018

Accepted 23rd July 2018

DOI: $10.1039 / c 8 r a 05606 d$

rsc.li/rsc-advances

\section{Recombinant human BMP-7 grafted poly(lactide- co-glycolide)/hydroxyapatite scaffolds via polydopamine for enhanced calvarial repair}

\begin{abstract}
Qinli Xu, Ye Li, Yuhang Zhu, Kunchi Zhao, Rui Gu* and Qingsan Zhu (DD*
Poly(lactic-co-glycolic acid) (PLGA) and hydroxyapatite (HA) are considered potential osteoinductive materials because of their biodegradability and mineralization features. However, the hydrophobicity of scaffold surfaces is less supportive of cell attachment and proliferation because of poor wettability. The mode of binding of growth factors to the scaffold also affects cell differentiation into osteoblasts. The half-life of a growth factor in vivo can be increased by binding the factor to the scaffold surface. In this work, we prepared a porous PLGA/HA scaffold grafted with recombinant human bone morphogenic protein-7 (rhBMP-7) attached via polydopamine (pDA) for bone repair. The pDA coated PLGA/HA (pDAPLGA/HA) scaffolds were characterized by energy dispersive $X$-ray analysis and Fourier-transform infrared spectroscopy. The microstructure and porosity of PLGA/HA scaffolds were analyzed by scanning electron microscopy and micro-computed tomography. The release profile of rhBMP-7 grafted onto the pDA-PLGA/HA (pDA-PLGA/HA/BMP-7) scaffolds was examined for 21 days. The attachment efficiency, cell proliferation rate, alkaline phosphatase activity, calcium deposition, and osteoblast-related gene expression of bone marrow-derived stem cells to PLGA/HA, pDA-PLGA/HA, and PDA-PLGA/HA/BMP-7 scaffolds were evaluated. To assess the ability of bone repair in vivo, scaffolds were implanted into critical-sized calvarial defects created in mice, and the in vivo tissue-engineered bone was monitored by micro-computed tomography and histology. In vivo experiments revealed rapid healing of the defects treated with the pDA-PLGA/HA/BMP-7 scaffolds compared with PDA-PLGA/HA and PLGA/HA scaffolds at week 8 post-surgery. These results collectively demonstrate that the rhBMP-7-immobilized PLGA/HA scaffold via pDA is a promising candidate for calvarial repair.
\end{abstract}

\section{Introduction}

Bone tissue engineering is a promising therapeutic strategy for healing bone defects caused by traumatic bone injury, tumor resection, congenital malformation, or other bone diseases. Autologous or allogenic bone grafting is most commonly applied in these cases. However, various limitations are inevitable, such as additional surgical invasion at a donor site, limited quantity of grafts, and disease transmission. ${ }^{\mathbf{1 , 2}}$ Bone regenerative medicine is a promising alternative to the conventional use of bone grafts. Over the past decades, various bone regeneration approaches have been studied including tissue engineering, biomaterials, stem cells, and drug screening.,

Over the last decade, poly(lactic-co-glycolic acid) (PLGA), hydroxyapatite (HA), and the combination of both have been used extensively as artificial scaffolds for bone repair. ${ }^{5-7} \mathrm{HA}$ is an inherent component of natural bone and has been widely used as a representative inorganic biomaterial for bone regeneration because of its good biocompatibility and

Department of Orthopedics, China-Japan Union Hospital, Jilin University, Changchun, PR China.E-mail: zhuqs@jlu.edu.cn; ccgurui@hotmail.com osteoconductivity. ${ }^{5-8}$ PLGA is a biodegradable polymer that is widely used as a tissue engineering scaffold material to build artificial bone, as a drug delivery carrier, and to create absorbable sutures. These uses reflect PLGA attributes of low immunogenicity, good biocompatibility, and suitable mechanical properties. $^{9-13}$ These advantages have generated interest in the use of PLGA/HA composite in material fabrication for bone repair. However, synthetic polymer scaffolds possess insufficient osteoinductivity due to the lack of osteoinductive factors. Surface modification of synthetic polymer scaffolds with osteoinductive biomolecules can guide cellular responses towards new bone formation, which is a promising approach to compensate for the lack of osteogenic potential of the pure scaffolds.

Bone morphogenic proteins (BMPs) can induce both osteoblast and chondrocyte differentiation, and thus can promote bone regeneration by enhancing both intramembranous and endochondral ossification. ${ }^{14}$ BMP-7 has been evaluated in small and large animal models, using both direct and indirect means of administration. Two parallel studies showed accelerated fracture healing in goats treated with BMP-7. ${ }^{15,16}$ Implantation of BMP-7 viral particles in dogs increased bone formation in mandibular 
defects. ${ }^{17}$ Recombinant human BMP-2 and BMP-7, which can be directly delivered, are the only osteoinductive molecules approved by the United States Food and Drug Administration for human applications. BMP-7 was approved for use in the U.S. in 2001 , followed by BMP-2 in 2004. The use of BMP-7 was first reported in humans undergoing tibial osteotomies. ${ }^{18}$

To achieve desired surface modification of synthetic scaffolds with osteoinductive biomolecules, a wide variety of immobilization techniques have been explored. They result in the formation of either covalent or non-covalent bonds between the biomolecules and the biomaterial surfaces. Dopamine is an adhesive protein of mussels that readily polymerizes to form a polydopamine (pDA) coating on the surfaces of a variety of wet materials. ${ }^{\mathbf{1 9}, 20}$ This simple one-step method has many advantages over other traditional material surface modification methods that require multistep procedures or special facilities, and it maintains the structure of the scaffold..$^{21,22}$ Furthermore, pDA coating can improve the hydrophilic character of the coated material and functionalize material surfaces. ${ }^{21-23}$ The adhesion and proliferation of osteoblasts is significantly promoted by pDA. Rim et al. ${ }^{24}$ fabricated the hydrophilic polylactic acid (PLLA) fibers modified by pDA coating. The pDA coated PLLA fibers were highly effective in inducing osteogenic differentiation and calcium mineralization of human mesenchymal stem cells. In addition, pDA can be used as a coupling agent to immobilize biomolecules onto the surface of polymer materials. Lee et al. ${ }^{25}$ successfully immobilized bone forming peptide-1 derived from BMP-7; the resulting fibrous scaffolds exhibited excellent bioactivities to support the adhesion and proliferation. Therefore, surface modification via pDA is an effective strategy to immobilize growth factors onto the surface of polymer materials.

Herein, we designed a bio-active scaffold using a simple and direct method to manufacture pDA-coated three-dimensional (3D) PLGA/HA porous scaffold grafted with recombinant human (rh)BMP-7 to promote bone regeneration (Scheme 1). The goal of this study was to effectively deliver rhBMP-7 in a sustained manner via pDA chemistry to create a favorable environment for cellular proliferation and osteogenic differentiation in vitro and in vivo.

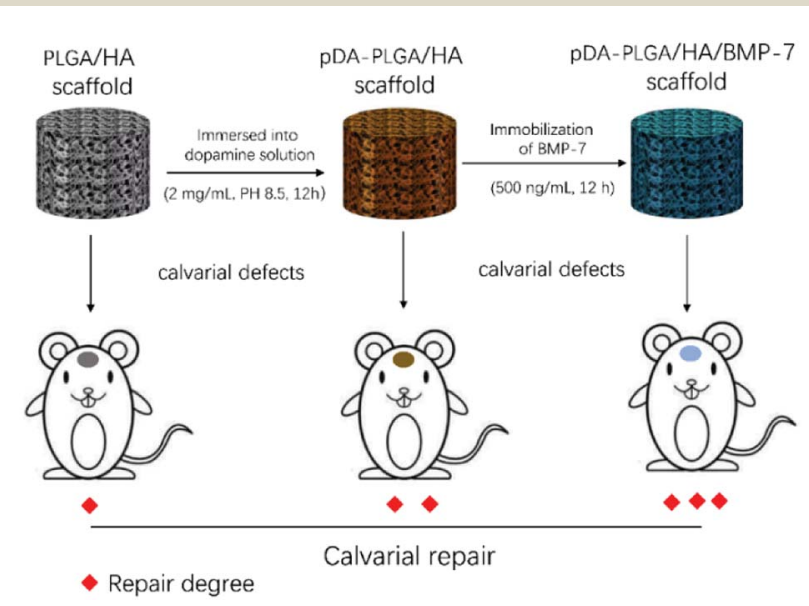

Scheme 1 Schematic illustration of establishment of the scaffoldmediated defect repair and evaluation of repair.

\section{Materials and methods}

\subsection{Preparation of porous PLGA/HA scaffolds}

The PLGA/HA composite was prepared with $10 \mathrm{wt} \%$ of HA (Aladdin, Shanghai, China, $M_{\mathrm{w}}=502.31$ ). PLGA/HA scaffolds were fabricated by combining phase separation and particulate leaching. Two grams of PLGA (LA : GA = 50:50, $14000 \mathrm{kDa}$; Jinan Daigang Biomaterial Co., Ltd., Jinan, China) was added to $10.0 \mathrm{~mL}$ of $N$-methyl pyrrolidone (NMP) (Sigma-Aldrich Co., Shanghai, China). The mixture was continuously stirred until the polymer was thoroughly dissolved. Sieved sodium chloride particulates $200-500 \mu \mathrm{m}$ in diameter were added to the PLGA solution. The weight ratio of the salt particulates to the polymer composites was $6: 1$. The mixture was cast in a homemade glass cylinder with a removable bottom. To remove NMP and salt particulates, the bottom was removed and the mixture was immersed in distilled water for 5 days with the water exchanged every $6 \mathrm{~h}$. Subsequently, any water remaining in the scaffolds was exchanged by ethanol. Finally, the porous PLGA/HA scaffolds were obtained after 3 days of lyophilization.

\section{2 pDA coating}

PLGA/HA scaffolds were immersed in a dopamine (Aladdin, Shanghai, China) solution ( $2 \mathrm{mg} \mathrm{mL}^{-1}$ in $10 \mathrm{mM}$ Tris- $\mathrm{HCl}, \mathrm{pH}$ $8.5)$ and incubated on a shaker for $12 \mathrm{~h}$ at room temperature $(24$ $\left.{ }^{\circ} \mathrm{C}\right)$. The pDA-coated scaffolds were then rinsed three times with deionized water to remove unattached dopamine.

\subsection{Characterization of morphology and chemical composition}

Surface morphology of the scaffolds was observed by scanning electron microscopy (SEM) using an XL30 ESEM-FEG device (FEI, USA). The scaffold was scanned by micro-computed tomography (micro-CT) using a SkyScan1076 apparatus (Bruker, Konitch, Belgium). Scan settings were voltage, $40 \mathrm{kV}$; current, $250 \mu \mathrm{A}$; integration time, $80 \mathrm{~ms}$; and isotropic voxel resolution, $16 \mu \mathrm{m}$. The scanned two-dimensional (2D) images were imported into software to produce three-dimensional (3D) formats through semi-automatic contouring and global thresholding. Appropriate gray values (100-150 pixels) were chosen to reconstruct the 3D distributions of PLGA/HA scaffolds. The chemical composition of the scaffolds was determined by energy-dispersive X-ray spectroscopy (EDX) and Fourier transform infrared spectroscopy (FTIR). The latter was done using an FTIR-2000 device (Perkin Elmer, USA).

\subsection{Evaluation of hydrophilicity of scaffolds}

The contact angle of static water was measured to evaluate the surface wettability of scaffolds using a DSA 100 Mk 2 goniometer (Kruss GmbH, USA). To estimate the amount of water uptake, dried samples were initially weighed and then immersed in a $10 \mathrm{~mL}$ vial containing distilled water. At predetermined times of 10, 30, and $60 \mathrm{~s}$, the samples were weighed after removing the surface water using a Kim wipe. The water uptake (\%) was calculated as follows: 
Water uptake $=\left[\left(W_{\text {after }}-W_{\text {before }}\right) / W_{\text {before }}\right] \times 100 \%$

\subsection{Binding and release analyses of rhBMP-7 with scaffolds}

pDA-PLGA/HA and PLGA/HA scaffolds were immersed in rhBMP-7 solutions at a concentration of $500 \mathrm{ng} \mathrm{mL}^{-1}$ at $4{ }^{\circ} \mathrm{C}$ for $12 \mathrm{~h}$. PLGA/HA scaffolds immersed in PBS at the same conditions were used as a control. All scaffolds were extensively washed with PBS $(5 \min \times 3)$ and then incubated with rabbit anti-mouse rhBMP-7 antibody ( $1: 2000$ dilution) for 2 hours at room temperature. After washing with PBS, scaffolds then incubated with a secondary Alexa Fluor 488 labeled goat antirabbit IgG antibody ( $1: 5000$ dilution) for $2 \mathrm{~h}$ at room temperature $\left(24^{\circ} \mathrm{C}\right)$ in the dark. Fluorescence images representative of the binding activities of PDA-PLGA/HA and PLGA/HA scaffolds were captured by the Maestro fluorescence imaging device (CRI, Germany).

pDA-PLGA/HA and PLGA/HA scaffolds were immersed in 500 ng $\mathrm{mL}^{-1}$ rhBMP-7 solution to obtain pDA-PLGA/HA/BMP-7 and PLGA/HA/BMP-7 scaffolds, which were used for the in vitro release study. Scaffolds were incubated in $2.0 \mathrm{~mL}$ PBS at $37^{\circ} \mathrm{C}$ for up to 21 days under orbital shaking with $100 \mathrm{rpm}$. At specified times, $0.2 \mathrm{~mL}$ of the supernatant was collected. The content of protein was measured by ELISA (R\&D). The release profiles were obtained by plotting the percentage of cumulatively content of released protein against time. The experiments were performed in triplicate.

\subsection{Cell adhesion and proliferation assays}

For the cell attachment efficiency assay, $1 \times 10^{5}$ bone marrowderived stem cells (BMSCs) were seeded onto the scaffolds (5 $\times 5 \times 3 \mathrm{~mm}^{3}$ ). At 3,6 and $12 \mathrm{~h}$, the culture medium was discarded and the unattached cells were washed off with PBS. The cells attached to the scaffolds were detached by trypsin/EDTA and enumerated using a blood counting chamber. The cell attachment efficiency was calculated as $N_{1} / N_{0}$, where $N_{1}$ and $N_{0}$ are the number of attached and seeded cells, respectively.

Cell proliferation was determined on day 1,3 , and 7 . The scaffolds were incubated in a solution of 3-(4,5-dimethylthiazol2-yl)-2,5-diphenyltetrazolium bromide (MTT, Aladdin, Shanghai, China; $5.0 \mathrm{mg} \mathrm{mL}^{-1}$ in PBS) for $4 \mathrm{~h}$. After the removal of the MTT solution, the acidified isopropanol $(0.2 \mathrm{~mL}$ of $0.04 \mathrm{M}$ $\mathrm{HCl}$ in $10 \mathrm{~mL}$ of isopropanol) was added to solubilize the resultant formazan product. The absorbance of the extractant at $492 \mathrm{~nm}$ was recorded using an Infinite M200 multifunction microplate scanner (Tecan, Switzerland). The result of the PLGA/HA scaffold group on day 1 was taken as the negative control with $100 \%$ viability. The cell proliferation rates (\%) of different scaffolds were calculated by absorbance [abs] different scaffolds/[abs] negative control $\times 100$.

\subsection{Cell differentiation assays}

Alkaline phosphatase (ALP) activity was determined after culturing BMSCs on different scaffolds in Dulbecco's modified Eagle's medium (DMEM)/F12 (Gibco) containing 10\% v/v fetal bovine serum (FBS) (Gibco) for 5 and 10 days. The medium of each well was carefully removed and the cells were washed three times with PBS, lysed in radio immunoprecipitation assay (RIPA) (Solarbio) buffer, frozen at $-80{ }^{\circ} \mathrm{C}$ for $30 \mathrm{~min}$, and thawed at $37{ }^{\circ} \mathrm{C}$. Then, $p$-nitrophenol phosphate substrate ( $p$ NPP) solution (Aladdin, Shanghai, China) was added and the samples were incubated in the dark for $30 \mathrm{~min}$ at $37^{\circ} \mathrm{C}$. The reaction was terminated with $3.0 \mathrm{M} \mathrm{NaOH}$ and the optical density (OD) values were measured with a multifunction microplate scanner at $405 \mathrm{~nm}$. The relative ALP activity was expressed as the OD value.

Calcium deposition was determined by Alizarin red S (ARS) (Aladdin, Shanghai, China) staining of the BMSCs after culture in DMEM/F12 containing 10\% v/v FBS for 14 and 21 days. After rinsing in water three times, the scaffolds were incubated in ARS stain solution (0.1\% ARS in Tris-HCl buffer, $\mathrm{pH}$ 8.0, SigmaAldrich, Shanghai, China) for $30 \mathrm{~min}$ at $37^{\circ} \mathrm{C}$. The scaffolds were then washed in distilled water for three times. The stained samples were treated with $10 \%(\mathrm{w} / \mathrm{v})$ cetylpyridinium chloride in $10 \mathrm{mM}$ sodium phosphate for $15 \mathrm{~min}$ at room temperature. The absorbance of ARS at $540 \mathrm{~nm}$ was recorded on a multifunction microplate scanner.

\subsection{Expression of osteogenic gene markers}

To evaluate the osteoinductive capacity of pDA-PLGA/HA/BMP7, pDA-PLGA/HA, and PLGA/HA scaffolds, osteosis-related genes were determined on day 3 and 7. The total RNA of BMSCs was extracted using Trizol (Invitrogen, Carlsbad, CA, USA). The concentration and purity of RNA were measured by the nanodrop One (Thermo Fisher Scientific, Waltham, MA, USA). The cDNA was synthesized using a ProtoScript cDNA Kit (NEB, Ipswich, MA, USA) according to protocols. Osteosisrelated genes (COL-I, RUNX-2, OPN, and OCN) primers were synthesized (Sangon Biotech, Shanghai, China) as summarized in Table $1 .{ }^{26}$ Gene amplification was performed as the description of Luna Universal qPCR Master Mix (NEB) protocol using a real-time PCR instrument (ABI 7500; Applied Biosystems, Franklin Lakes, NJ, USA). The initial heating at $95{ }^{\circ} \mathrm{C}$ for $60 \mathrm{~s}$ was followed by 45 cycles at $95{ }^{\circ} \mathrm{C}$ for $15 \mathrm{~s}$ and $60{ }^{\circ} \mathrm{C}$ for $30 \mathrm{~s}$. Melt curve analysis was performed to measure the reliability of qPCR. Each sample was tested in triplicate.

\subsection{In vivo surgery and animal care}

A combination of ketamine $\left(135 \mathrm{mg} \mathrm{kg}^{-1}\right)$ and xylazine $(15 \mathrm{mg}$ $\mathrm{kg}^{-1}$ ) was administered intraperitoneally to anesthetize the mice. An incision was made to expose the cranium and a $4 \mathrm{~mm}$ critical-sized defect was made on the right side using a drill bit, taking care that the underlying dura mater was not damaged. A one-hole mouse calvarial model was used and the scaffolds were placed into these defects as per the study design. Finally, the periosteum, muscles, and skin were sutured separately for closure. Rats possessed free access to food and water after surgery. Penicillin was injected intramuscularly $(10000 \mathrm{U}$ day $^{-1}$ ) beginning immediately after surgery and for 3 days thereafter. All animal studies were conducted in accordance with the principles and procedures outlined in "Regulations for 
Table 1 Genes and Primer Nucleotide Sequences

\begin{tabular}{llll}
\hline Gene & Primer sequence $\left(5^{\prime}-3^{\prime}\right)$ & Length (bp) & Reference \\
\hline COL-I & F: CGCTGGCAAGAATGGCGATC & 21 & NM_007742.3 \\
& R: ATGCCTCTGTCACCTTGTTCG & 24 & NM_001145920.1 \\
RUNX-2 & F: GCCCTCATCCTTCACTCCAAG & 21 & 21 \\
OPN & R: GGTCAGTCAGTGCCTTTCCTC & 21 & NM_007742.3 \\
& F: TCAGGACAACAACGGAAAGGG & 21 & L24431.1 \\
OCN & R: GGAACTTGCTTGACTATCGATCAC & 24 & 21 \\
GAPDH & F: AGCAGGAGGGCAATAAGGTAG & 20 & NM_008084.2
\end{tabular}

the Administration of Affairs Concerning Laboratory Animals", approved by the National Council of China on October 31, 1988 and "The National Regulation of China for Care and Use of Laboratory Animals", promulgated by the National Science and Technology Commission of China, on November 14, 1988 as Decree No. 2. Protocols were approved by the Committee of Jilin University Institutional Animal Care and Use.

\subsection{Histological evaluation}

Eight weeks after the operation, the animals were killed. All specimens were prepared for histological evaluation. Specimens were decalcified for 15 days with 15\% ethylenediaminetetraacetate (EDTA)-buffered saline solution, dehydrated with gradient ethanol solutions, embedded in paraffin blocks, and sectioned perpendicularly to their longitudinal axis into $5 \mu \mathrm{m}$-thick slices. Masson trichrome and hematoxylin and eosin (H\&E) were used to stain these slices. The histology slide images were acquired were scanned with an Aperio tissue scanner (ScanScope XT, USA).

\subsection{Micro-CT evaluation}

The neo-bone formation was analyzed by micro-CT. Approximately 160 projections were acquired over a rotation range of $180^{\circ}$, with a $4^{\circ}$ rotation step. The full length of each bone was scanned, and, on average, consisted of 800 slices. 3D virtual models of representative regions in the scaffolds were created and visualized using MIMICS 16.0 software (Materialise's interactive medical image control system; Leuven, Belgium). The visualized 3D images were shown in the gross profiles including trabecular and cortical bone ranges. The density of newly formed bone was determined by assigning a threshold for total bone mineral content within the initial defect and subtracting any contribution from the scaffold. The extent of the neo-bone formation in micro-CT was calculated by the relative ratio of newly formed bone mineral density determined during the 8 weeks experiment.

\subsection{Mechanical push-out testing}

At 2, 4, and 8 weeks after surgery, the animals were killed. Specimens were stored in buffered formalin solution and refrigerated until testing. Specimens were allowed to equilibrate to room temperature before testing was conducted. Calvaria specimens were placed on the fixture platform, resting the superior face of the calvarium on the platform and centering the circular defect of the dural face with the indenter axis. Care was also taken to align the face of the defect to be parallel to the indenter face. Under displacement control, the indenter was forced into the repair zone surface at a rate of $5 \mathrm{~mm} \mathrm{~min}^{-1}$ until failure and was continued until total displacement occurred.

\subsection{Statistical analysis}

The data are presented as mean \pm standard deviation (SD). The independent and replicated experiments were used to analyze the statistical variability of the data analyzed using Student's $t$ test, and $p<0.05$ was considered to be significant.

\section{Results}

\subsection{SEM, micro-CT, contact angle, and water uptake}

The macroscopic morphology and microstructure of solidified composites by phase separation were evident by SEM and micro-CT. After incubating in the dopamine solution, the color of scaffolds changed from white to dark brown, indicating catechol oxidation and subsequent dopamine polymerization (Fig. 1a). Scaffolds possessed microchannels with an average diameter of 300-400 $\mu \mathrm{m}$ (Fig. 1b). These microchannels presented a regularly roughened surface (Fig. 1c). To evaluate the internal microstructure and porosity of PLGA/HA scaffold, micro-CT was used to scan the PLGA/HA scaffold. The 3D reconstructions and axial images are displayed in Fig. 1d and e. In the axial view, a large number of pore structures were observed to be uniformly distributed within the PLGA/HA scaffold. The total porosity of the PLGA/HA scaffold reached 74\% as determined using CT Analyzer Version: 1.15.4.0 microCT analysis software. Subsequently, the PLGA/HA scaffolds were surface modified using pDA chemistry (Fig. 2a and b). Fig. 2a shows the results of the water uptake experiment as performed against PLGA/HA and pDA-PLGA/HA scaffolds. Unmodified PLGA/HA scaffolds showed a very poor water uptake rate over $60 \mathrm{~s}$. However, the pDA-PLGA/HA scaffolds rapidly absorbed water. Additionally, the pDA-PLGA/HA scaffolds sank in the cell culture medium. However, PLGA/HA scaffolds floated on the surface. These results were also confirmed in contact angle analysis. The water contact angle was $75.3^{\circ}$ for the PLGA/HA scaffolds and $27.2^{\circ}$ for the pDAPLGA/HA scaffolds (Fig. 2b). This was due to the hydrophilic pDA coated on the surface of scaffolds. 


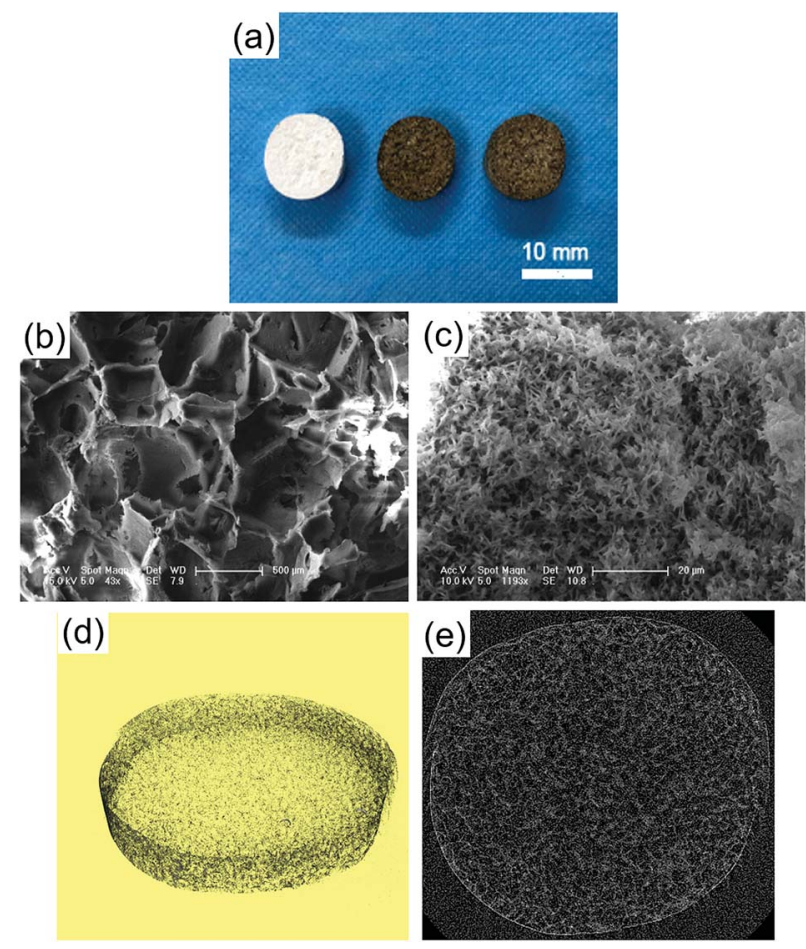

Fig. 1 Macroscopic morphology of PLGA/HA, pDA-PLGA/HA, and pDA-PLGA/HA/BMP-7 scaffolds (a). SEM images of internal structures for porous PLGA/HA scaffold ( $b$ and $c$ ). Micro-CT 3D reconstruction of PLGA/HA scaffold (d) and the axial image (e).

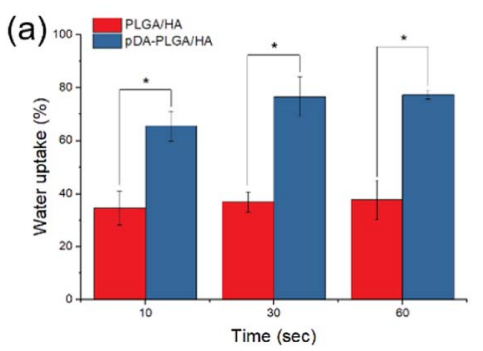

(b)

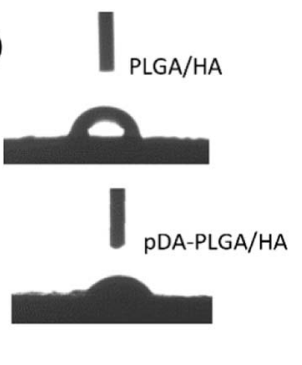

Fig. 2 Water uptake rate (a) and initial contact angle (b) of PLGA/HA and PDA-PLGA/HA scaffolds. $(n=3)$.

\subsection{FTIR and EDX analyses}

EDX and FTIR were used to determine the surface chemical property of the scaffolds. The EDX spectra detected the presence of carbon $(\mathrm{C})$ and oxygen $(\mathrm{O})$ elements. A calcium $(\mathrm{Ca})$ peak was also observed, which demonstrated that HA was exposed on the surface of scaffolds. After pDA coating, new nitrogen (N) peaks were found on the EDX spectra of scaffolds. FTIR spectra also showed the pDA coating. An absorption band typically appeared at $2990 \mathrm{~cm}^{-1}$ and $2940 \mathrm{~cm}^{-1}$ (-CH3), $1753 \mathrm{~cm}^{-1}(\mathrm{C}=\mathrm{O})$, and $1183 \mathrm{~cm}^{-1}$ and $1083 \mathrm{~cm}^{-1}(\mathrm{C}=\mathrm{O})$ (Fig. 3). The band characteristic for HA was observed at $500-600 \mathrm{~cm}^{-1}(\mathrm{P}=\mathrm{O})$, which clearly suggested the presence of HA in scaffolds. After pDA coating, new bands were observed at $1632 \mathrm{~cm}^{-1}$ and 3000$3600 \mathrm{~cm}^{-1}$ suggesting the presence of pDA on the surface of scaffolds. These FTIR spectra revealed that pDA was

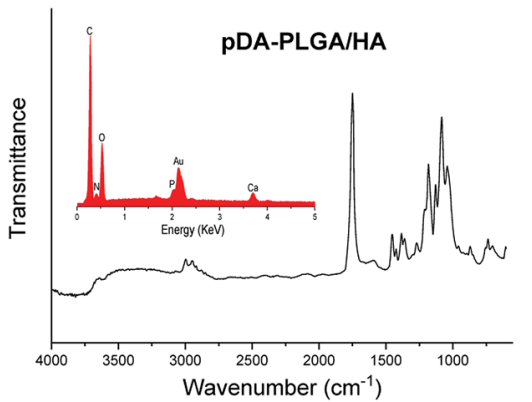

Fig. 3 FTIR and EDX analyses of pDA-PLGA/HA scaffolds.

successfully coated on PLGA/HA porous scaffolds. Our results are consistent with several previous reports. ${ }^{27,28}$

\section{3 rhBMP-7 binding ability with scaffolds and rhBMP-7 release kinetics from scaffolds}

The binding capacity of each scaffold with rhBMP-7 was evaluated with an immunofluorescence assay using a monoclonal anti-rhBMP-7 antibody and a secondary AlexaFluor 488-labeled anti-mouse IgG antibody (Fig. 4a). The fluorescence signal of pDA-PLGA/HA scaffold was higher than that of the PLGA/HA scaffold and the control. These results indicated that the amount of rhBMP-7 on the surface of the pDA-PLGA/HA (Avg Signal $=1223$ ) scaffold was higher than that of the PLGA/HA $($ Avg Signal $=428)$ scaffold, which was mainly due to the binding ability of pDA.

We performed a release test of rhBMP-7 from the pDA-PLGA/ HA/BMP-7 and PLGA/HA/BMP-7 scaffolds. The pDA-PLGA/HA/ BMP-7 scaffolds exhibited a sustained release profile for up to 21 days without burst release (Fig. 4b). Over the 21 days study,

(a)

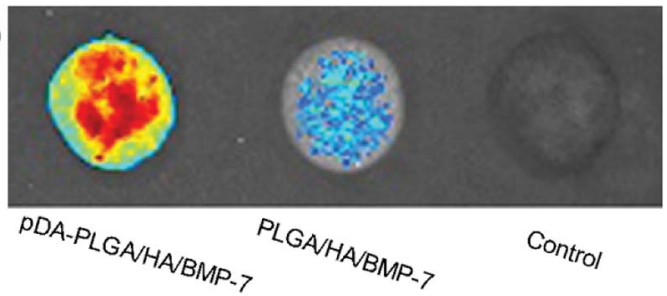

(b)

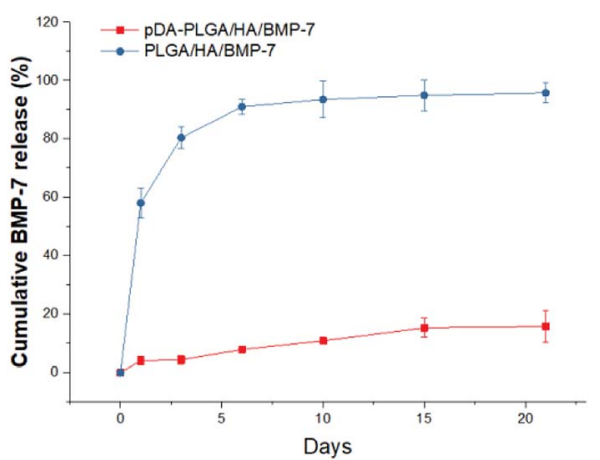

Fig. 4 (a) rhBMP-7 binding ability of pDA-PLGA/HA and PLGA/HA scaffolds were detected by immunofluorescence. (b) Release kinetics of rhBMP-7 from pDA-PLGA/HA/BMP-7 and PLGA/HA/BMP-7 scaffolds $(n=3)$. 
only $15.8 \%$ of the rhBMP-7 load was released from the pDAPLGA/HA/BMP-7 scaffolds. On the contrary, 90\% of the BMP-7 was released in the first 6 days from the PLGA/HA/BMP-7 scaffolds. These results clearly showed that BMP-7 was stably immobilized on the surface of the pDA-PLGA/HA/BMP-7 scaffolds.

\subsection{Cell adhesion, proliferation, and differentiation in scaffolds}

The attachment efficiency of BMSCs cultured on various scaffolds for 3, 6 and $12 \mathrm{~h}$ are summarized in Fig. 5a. After cultured for $3 \mathrm{~h}$, the cell attachment efficiency on the pDA-PLGA/HA and pDA-PLGA/HA/BMP-7 scaffolds reached more than 30\%, which was significantly greater than the percentage on the PLGA/HA scaffolds $(12.5 \%)$. When cultured for $6 \mathrm{~h}$, the cell attachment efficiency on the pDA-PLGA/HA/BMP-7 and pDA-PLGA/HA scaffolds increased to approximately $85 \%$, which was greater than the $44.6 \%$ observed for the PLGA/HA scaffolds. BMSC proliferation was quantitatively monitored using the MTT assay to measure the metabolic activity of the total population of cells for 1, 3, and 7 days. As shown in Fig. 5b, each scaffold supported the proliferation of BMSCs within 7 days. The pDA-PLGA/HA/ BMP-7 scaffold exhibited the highest cellular activity, whereas the PLGA/HA scaffold showed the lowest at 3 and 7 days, suggesting that the pDA and rhBMP-7 coating promoted cell attachment and proliferation.

To investigate the osteogenic differentiation of BMSCs on different scaffolds, ALP activity and calcium deposition were measured. Fig. 6a shows the ALP activities of the BMSCs cultured on the different scaffolds after 5 and 10 days. The ALP activity detected in cells cultured on the pDA-PLGA/HA/BMP-7 scaffold was significantly higher than those on the PLGA/HA and pDA-PLGA/HA scaffolds ( $p<0.05$ for both). Calcium deposition was measured by ARS. As shown in Fig. 6b-e, quantification of ARS indicated that the deposition of calcium minerals in the pDA-PLGA/HA/BMP-7 scaffolds were significantly higher than those in the other scaffolds $(p<0.05)$. Moreover, after 21 days of culture, the deposition of calcium minerals in the pDAPLGA/HA/BMP-7 scaffolds were greater than those in the pDAPLGA/HA and PLGA/HA scaffolds $(p<0.05)$.

\subsection{Gene expression studies by Q-PCR}

At days 3 and 7, total RNA was isolated and then used for realtime PCR analysis of osteosis-related genes (Fig. 7). On day 3, the expression of COL-I, RUNX-2, and OCN in BMSCs from pDA- (a)

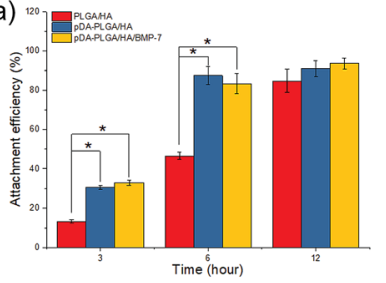

(b)

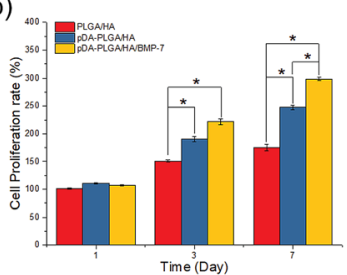

Fig. 5 In vitro cell adhesion and proliferation in the scaffolds of PLGA/ HA, pDA-PLGA/HA and pDA-PLGA/HA/BMP-7. $(n=3)$.

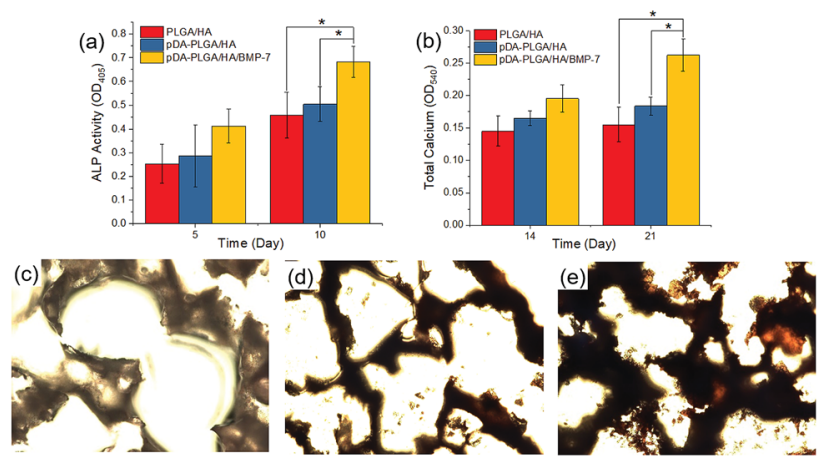

Fig. 6 ALP activities (a), calcium deposition (b) and alizarin red $S$ staining at 21 days for PLGA/HA (c), pDA-PLGA/HA (d) and pDA-PLGA/ HA/BMP-7 (e) scaffolds. $(n=3)$.

PLGA/HA/BMP-7 scaffolds were up-regulated compared with PLGA/HA and pDA-PLGA/HA scaffolds. COL-1 expression in pDA-PLGA/HA/BMP-7 group was 2.87 and 8.65 times higher than those in pDA-PLGA/HA and PLGA/HA groups, respectively. RUNX-2 expression in pDA-PLGA/HA/BMP-7 group was 2.61 and 5.35 times higher than those in pDA-PLGA/HA and PLGA/HA groups, respectively. OCN expression in pDA-PLGA/HA/BMP-7 group was 1.92 and 3.13 times higher than those in pDAPLGA/HA and PLGA/HA groups, respectively. OPN expression in pDA-PLGA/HA/BMP-7 group was slightly lower than that in PDA-PLGA/HA group. On day 7, expressions of Col-I, RUNX-2, OPN and OCN in cells from pDA-PLGA/HA/BMP-7 Scaffolds were significantly higher than those from pDA-PLGA/HA scaffolds. The expressions of Col-I, RUNX-2, OPN and OCN in the pDA-PLGA/HA/BMP-7 group were 1.48-, 1.67-, 3.35-, and 1.45fold higher than those in the pDA-PLGA/HA group.

\subsection{In vivo histological evaluation}

Bone formation was further analyzed histologically with $\mathrm{H} \& \mathrm{E}$ and Masson Trichrome staining. As shown in Fig. 8a-d, no obvious sign of inflammation or severe immunological
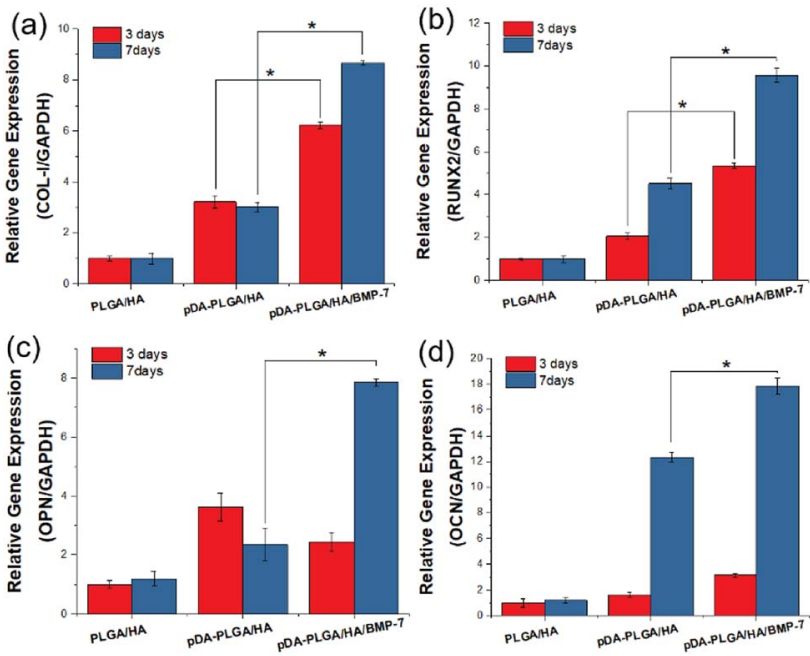

Fig. 7 Expression of COL-I (a), RUNX-2 (b), OPN (c) and OCN (d) in BMSCs after 3 and 7 days. $(n=3)$. 

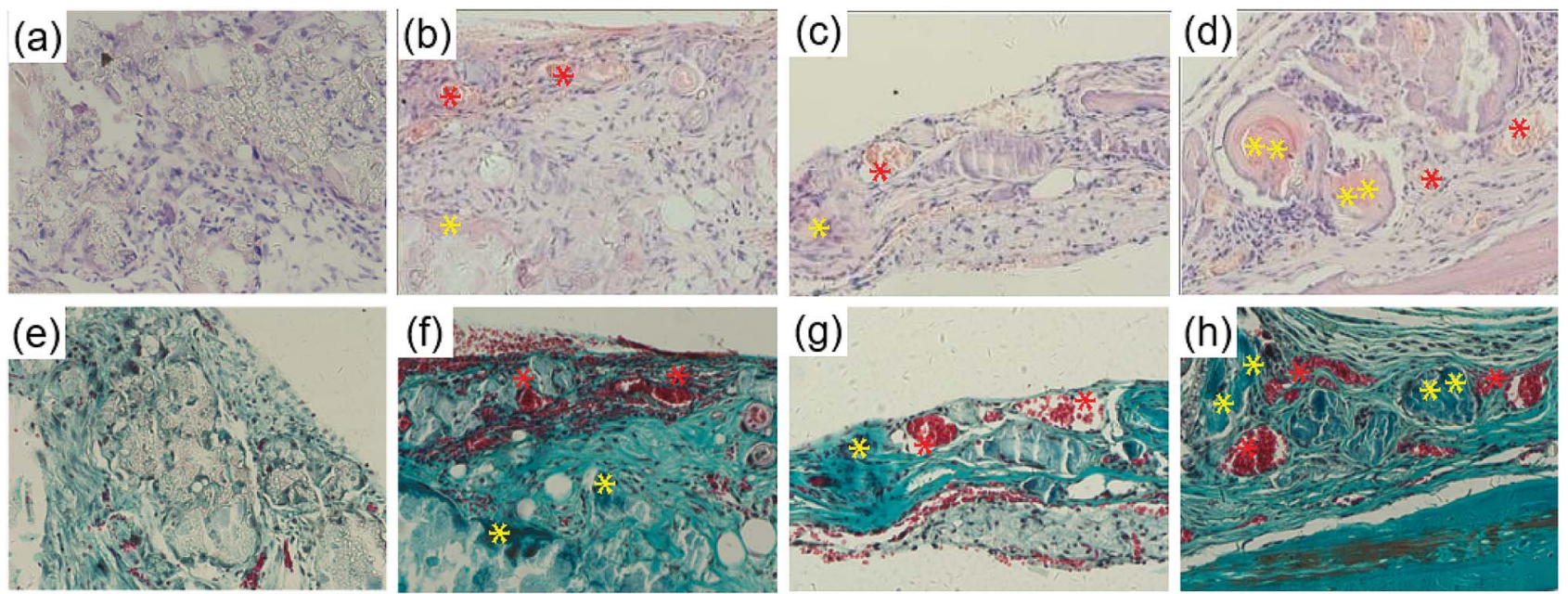

Fig. 8 H\&E (a-d) and Masson Trichrome (e-h) staining of the implants 8 weeks after operation, (a) and (e) are blank group, (b) and ( $f$ ) are PLGA/ HA group, (c) and (g) are pDA-PLGA/HA group, (d) and (h) are pDA-PLGA/HA/BMP-7 group. Red "*" indicates blood vessels and yellow "*" indicates new bone.
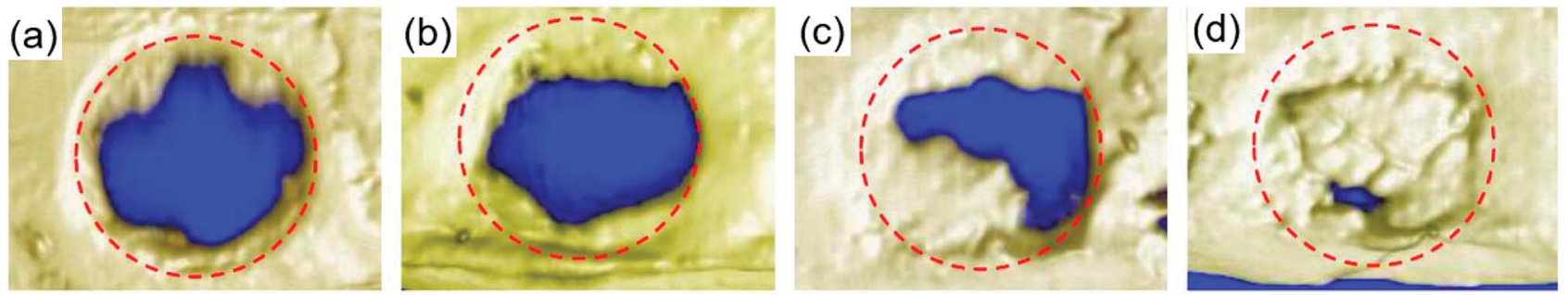

Fig. 9 Micro-CT analysis of calvarial defects. Micro-CT images showing the mineralization within a 4 mm calvarial defect either left empty with no scaffold implanted (a) blank or treated with (b) PLGA/HA, (c) pDA-PLGA/HA, and (d) pDA-PLGA/HA/BMP-7 scaffolds at week 8 post-surgery. The red breakpoint lines indicate the location of the original mouse calvarial defect.

response was observed in all groups. The blank group remained poorly repaired and was only filled with connective tissue at 8 weeks. The PLGA/HA scaffold group was almost degraded at 8 weeks with an immature bony bridge, the same as the pDAPLGA/HA group. In the pDA-PLGA/HA/BMP-7 group, the defect was repaired with thick new bone. Furthermore, blood vessels and trabeculae surrounded by osteoblasts were present. Masson staining confirmed the above results with a better exhibition of tissue microstructures and matrix components. Large defects remained unrepaired at 8 weeks in the blank group with blue-stained connective tissue filled chaotically (Fig. 8e-h). At 8 weeks, partial healing was evident in the PLGA/ HA and pDA-PLGA/HA scaffold groups. Superior outcome was confirmed in the pDA-PLGA/HA/BMP-7 group with the defect almost fully replaced by woven bone (blue stained). More mature arrangement of collagen could also be observed.

\subsection{Confirmation of bone formation by micro-CT}

For bone scaffold, micro-CT is often and widely used to quantify bone mineral densities of newly formed bones. Each experimental model was analyzed at week 8 after scaffold implantation. Fig. 9 shows the 3D micro-CT analysis, which revealed the changes in bone volume for the PLGA/HA, pDA-PLGA/HA and
pDA-PLGA/HA/BMP-7 groups compared to the blank group. Micro-CT data showed that $97.60 \%$ of pDA-PLGA/HA/BMP-7, $60.27 \%$ of PLGA/HA/BMP-7, and $39.11 \%$ of PLGA/HA of these defects were mineralized (Fig. 9b-d) compared to the blank defect (35.19\%). Compared to pDA-PLGA/HA group (60.27\%), a significant increase was observed as to the percentage of bone volume in pDA-PLGA/HA/BMP-7 group (97.60\%) (Fig. 9c and d).

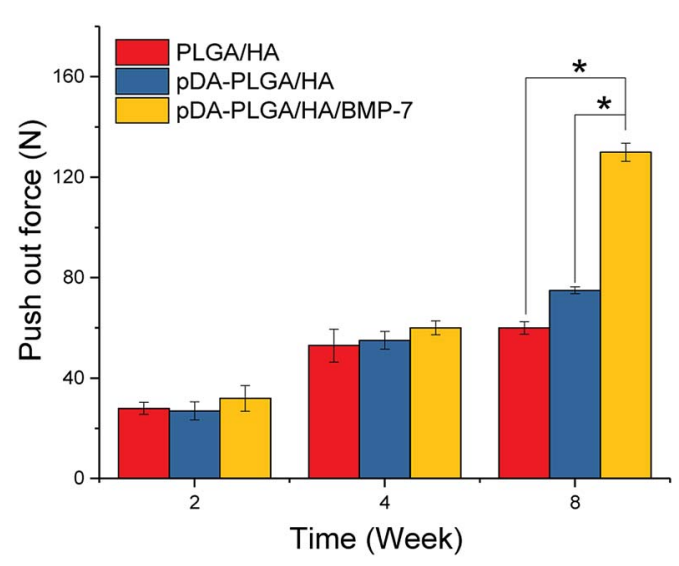

Fig. 10 Push-out strength of the calvarial regenerated bone. 
This was supported by the morphological appearance of the bone-like tissue, which formed within the defect when analyzed histologically (Fig. 8c, d, g, and f).

\subsection{In vivo push-out strength}

Functional testing of the regenerated bone strength was carried out by push-out testing. The pDA-PLGA/HA scaffolds group showed greater push-out strength than that of the uncoated group (Fig. 10). The push-out strength of the pDA-PLGA/HA/ BMP-7 scaffolds group was significantly higher than those of the groups without rhBMP-7. By comparison, the strength of the native intact calvarial controls was $340 \pm 52 \mathrm{~N}$.

\section{Discussion}

Biodegradable polymeric scaffolds used for bone reconstruction have received significant attention because of their ability to provide a spatially and temporally appropriate environment for new bone tissue growth. ${ }^{29,30}$ Polylactone-type biodegradable polymers, such as poly(L-lactide) (PLA), polyglycolide (PGA), and PLGA are commonly used for the preparation of scaffolds. PLGA is an FDA-approved material for scaffolds. Attributes of PLGA include low immunogenicity, non-toxicity, and adjustable degradation rate. However, these polymeric scaffolds impede cell attachment and penetration due to the lack of cell anchoring sites, and they have poor hydrophilicity and low surface energy. ${ }^{31}$ These properties can hinder cell adhesion and migration. In order to improve the hydrophilicity of PLGA, HA was added to PLGA matrix, which could effectively improve the hydrophilicity and promote cell adhesion and proliferation. ${ }^{32}$ To improve the situation, we introduced hydrophilicity by blending with HA and coating the PLGA/HA scaffolds using pDA chemistry to enhance the bone like-cell attachment and promote cellular migration (Fig. 5). Jo et al. ${ }^{33}$ demonstrated that bioplotted 3D PCL scaffolds modified with pDA chemistry displayed good infiltration of cells into the scaffolds, which facilitated cell proliferation and migration. Similarly, due to the pDA effect, our cell viability results indicated that the pDA-PLGA/HA scaffolds also showed enhanced cell proliferation as compared with pure PLGA/HA (Fig. 5). Surface wettability is an important factor for the function of mammalian cells as well as osteoblast adhesion on biomaterials. These parameters are crucial for bone tissue regeneration. ${ }^{34,35}$ EDX and FTIR results demonstrate that PDA was successfully coated onto the surface of PLGA/HA scaffolds. Static water contact angle determination and water uptake test results showed that the hydrophilicity of the scaffold was improved after the pDA treatment (Fig. 2). The pDA-coat substrate can also act as a conjugate to peptides and bioactive molecules containing primary amine and/or thiol groups via imine formation or Michaelis addition.

To accelerate the osteogenic activity in this study, we used rhBMP-7 to induce cellular differentiation for bone tissue regeneration. We chose rhBMP-7 because BMPs are multifunctional cytokines that belong to the transforming growth factor-beta superfamily of proteins and also play a crucial role in bone formation. BMPs are also approved by the FDA. ${ }^{36,37}$
Moreover, BMPs mediate cell proliferation, differentiation, and promote osteogenesis. ${ }^{38}$ BMPs are also an essential signaling molecule involved in the recovery of fractured bone. ${ }^{39,40}$ Additionally, Lee et $a .^{25}$ showed that pDA-coated PLGA electrospun nanofibers could immobilize bone forming peptide- 1 derived from bone morphogenetic protein-7 and promoted bone tissue regeneration. Ko et al. $^{\mathbf{4 1}}$ demonstrated that porous PLGA scaffolds could serve to conjugate osteoinductive biomolecules (such as rhBMP-2) via pDA chemistry. These scaffolds possessed greater bone regeneration than bare scaffolds. In the present study, we employed pDA chemistry for the effective delivery of rhBMP-7 with a sustained release pattern to promote bone tissue regeneration (Fig. 4b). Cell adhesion assay showed enhanced cell attachment to the scaffold surface as a result of the pDA coating. Cell proliferation assay indicated that rhBMP-7 was successfully conglutinated to the scaffold via pDA and accounted for the positive effect in cell proliferation (Fig. 5). There was a significant enhancement in osteogenetic differentiation with the rhBMP-7 loaded scaffold in cell differentiation assay (Fig. 6). Osteoblast-related genes (COL-I, RUNX-2, OPN and OCN) in the pDA-PLGA/HA group were up-regulated compared with those in the PLGA/HA group on days 3 and 7 (Fig. 7). This observation explains the influence of the scaffold surface morphology and chemistry on osteoblast differentiation and mineralization. A previous study demonstrated its beneficial effect on osteoblast differentiation in vitro. ${ }^{7}$ The coating of pDA on PLGA/HA scaffold surface could effectively improve hydrophilicity, cell adhesion, and the expression of osteoblastrelated genes. An additional influence of rhBMP-7 on BMSCs osteogenic differentiation was observed in the expression of osteoblast-related genes. rhBMP-7 signaling regulates processes in bone formation. RUNX-2 is needed for osteogenic differentiation and also plays an important role in proper function of mature osteoblasts, including the synthesis of bone matrix (COL-I). ${ }^{42}$ High expression of RUNX-2 is an important marker for BMSCs differentiation into osteoblasts. rhBMP-7 induces RUNX-2 expression in BMSCs and promotes osteoblastic differentiation. A significant increase in mineralization was also found in pDA-PLGA/HA/BMP-7 group, which can be attributed to the significant increase in the expression of OCN and OPN. These genes are usually regarded as late markers for osteoblast differentiation and play an important role in bone repair. ${ }^{\mathbf{4 3 , 4 4}}$

We created critical-sized defects in mice, applied three types of scaffolds to the defects, and extracted and analyzed calvarial bone specimens by histological evaluation and micro-CT (Fig. 8 and 9). Tissue sections from the defect-only group and the group implanted with PLGA/HA showed loosely-formed connected tissue. On the contrary, the portion of the mineralized collagen was qualitatively enhanced in the pDA-PLGA/HA and pDA-PLGA/HA/BMP-7 scaffold groups. Notably, the scaffold immobilized with rhBMP-7 were mostly collapsed and appeared to be degraded, with new and immature bone observed in close contact with the remnants of the degraded scaffolds. In these cases, bone formation was primarily induced by the implanted scaffolds, suggesting that the pore size of the scaffold is effective in promoting the penetration of tissue into the bone cavity. The results for bone formation were consistent in both micro-CT 
and histological analysis. The evaluation of newborn bone integration with host bone, which was shown in the pushingout test, further verified the micro-CT and histological outcome (Fig. 10).

When the bone defect area is especially large, additional bone grafts are required to accelerate bone regeneration before the disruption of the membranes. ${ }^{45}$ In this study, pDA-PLGA/ HA/BMP-7 scaffolds displayed many advantages including enhanced bone regeneration, and remarkable integration with the host tissue.

\section{Conclusions}

We fabricated porous PLGA/HA 3D scaffolds and immobilized rhBMP-7 with a simple pDA coating method. EDX and FTIR analyses demonstrated that the PLGA/HA scaffold was successfully coated by pDA chemistry, which improved the hydrophilicity. In vitro study showed that rhBMP-7 could be immobilized by pDA coating and released in a sustained manner. The introduction of rhBMP-7 on the surface of PLGA/HA scaffold via pDA can effectively promote the expression of osteoblast-related genes and enhance proliferation and differentiation of BMSCs. When scaffolds were implanted in calvarial critical-sized defects in mice for eight weeks, the best effect of bone regeneration was observed in pDA-PLGA/HA/rhBMP-7 group by histological and micro-CT analyses. These results collectively demonstrate that the rhBMP-7-immobilized PLGA/HA scaffold via pDA is a promising candidate for calvarial repair.

\section{Conflicts of interest}

There are no conflicts to declare.

\section{Acknowledgements}

This study was supported by Industrial Innovation Special Fund Project of Jilin Province, China (2018C005) and the crosswise tasks of Shanghai Sanyou Medical Co., Ltd (3R215P403430)

\section{References}

1 J. S. Silber, D. G. Anderson, S. D. Daffner, B. T. Brislin, J. M. Leland, A. S. Hilibrand, A. R. Vaccaro and T. J. Albert, Spine, 2003, 28, 134-139.

2 H. J. Mankin, F. J. Hornicek and K. A. Raskin, Clin. Orthop. Relat. Res., 2005, 210-216.

3 A. Khojasteh, F. Fahimipour, M. B. Eslaminejad, M. Jafarian, S. Jahangir, F. Bastami, M. Tahriri, A. Karkhaneh and L. Tayebi, Mater. Sci. Eng., C, 2016, 69, 780-788.

4 M. Ngiam, S. Liao, A. J. Patil, Z. Cheng, C. K. Chan and S. Ramakrishna, Bone, 2009, 45, 4-16.

5 X. Wang, G. Zhang, F. Qi, Y. Cheng, X. Lu, L. Wang, J. Zhao and B. Zhao, Int. J. Nanomed., 2018, 13, 117-127.

6 D. B. Bhuiyan, J. C. Middleton, R. Tannenbaum and T. M. Wick, Biomed. Mater. Eng., 2017, 28, 671-685.

7 X. Zhao, Y. Han, J. Li, B. Cai, H. Gao, W. Feng, S. Li, J. Liu and D. Li, Mater. Sci. Eng., C, 2017, 78, 658-666.
8 J. Zhang, H. Liu, J.-X. Ding, J. Wu, X.-L. Zhuang, X.-S. Chen, J.-C. Wang, J.-B. Yin and Z.-M. Li, ACS Biomater. Sci. Eng., 2016, 2, 1471-1482.

9 Y. C. Shin, W. J. Yang, J. H. Lee, J. W. Oh, T. W. Kim, J. C. Park, S. H. Hyon and D. W. Han, Int. J. Nanomed., 2014, 9, 4067-4078.

10 F. Sun, T. Shi, T. Zhou, D. Dong, J. Xie, R. Wang, X. An, M. Chen and J. Cai, J. Biomed. Nanotechnol., 2017, 13, 290302.

11 M. Bi, H. Han, S. Dong, Y. Zhang, W. Xu, B. Zhu, J. Wang, Y. Zhou and J. Ding, Polymers, 2018, 10, 109.

12 J. Zhang, S.-G. Yang, J.-X. Ding and Z.-M. Li, RSC Adv., 2016, 6, 47418-47426.

13 N. Zhang, Y. Wang, W. Xu, Y. Hu and J. Ding, Polymers, 2016, 8, 218.

14 J. Wang, D. Li, T. Li, J. Ding, J. Liu, B. Li and X. Chen, Materials, 2015, 8, 1009-1026.

15 F. C. den Boer, J. A. Bramer, T. J. Blokhuis, E. J. Van Soest, J. M. Jenner, P. Patka, F. C. Bakker, E. H. Burger and H. J. Haarman, Bone, 2002, 31, 158-164.

16 T. J. Blokhuis, F. C. den Boer, J. A. Bramer, J. M. Jenner, F. C. Bakker, P. Patka and H. J. Haarman, Biomaterials, 2001, 22, 725-730.

17 Y. Zhang, J. Song, B. Shi, Y. Wang, X. Chen, C. Huang, X. Yang, D. Xu and X. Cheng, Biomaterials, 2007, 28, 46354642.

18 K. D. Hankenson, K. Gagne and M. Shaughnessy, Adv. Drug Delivery Rev., 2015, 94, 3-12.

19 Y. Li, M. Qin, Y. Cao and W. Wang, Langmuir, 2014, 30, 43584366.

20 Y. Li, H. Liu, T. Wang, M. Qin, Y. Cao and W. Wang, ChemPhysChem, 2017, 18, 1466-1469.

21 F. Scognamiglio, A. Travan, M. Borgogna, I. Donati, E. Marsich, J. W. Bosmans, L. Perge, M. P. Foulc, N. D. Bouvy and S. Paoletti, Acta Biomater., 2016, 44, 232-242.

22 Y. M. Shin, Y. B. Lee, S. J. Kim, J. K. Kang, J. C. Park, W. Jang and H. Shin, Biomacromolecules, 2012, 13, 2020-2028.

23 S. J. Lee, D. Lee, T. R. Yoon, H. K. Kim, H. H. Jo, J. S. Park, J. H. Lee, W. D. Kim, I. K. Kwon and S. A. Park, Acta Biomater., 2016, 40, 182-191.

24 N. G. Rim, S. J. Kim, Y. M. Shin, I. Jun, D. W. Lim, J. H. Park and H. Shin, Colloids Surf., B, 2012, 91, 189-197.

25 Y. J. Lee, J. H. Lee, H. J. Cho, H. K. Kim, T. R. Yoon and H. Shin, Biomaterials, 2013, 34, 5059-5069.

26 Y. Wang, H. Cui, Z. Wu, N. Wu, Z. Wang, X. Chen, Y. Wei and P. Zhang, PLoS One, 2016, 11, e0154924.

27 Y. Li, Y. Shi, S. Duan, D. Shan, Z. Wu, Q. Cai and X. Yang, J. Biomed. Mater. Res., 2014, 102, 3894-3902.

28 T. Gao, N. Zhang, Z. Wang, Y. Wang, Y. Liu, Y. Ito and P. Zhang, Macromol. Biosci., 2015, 15, 1070-1080.

29 F. Shuang, S. X. Hou, Y. T. Zhao, H. B. Zhong, C. Xue, J. L. Zhu, G. Y. Bu and Z. Cao, Eur. Rev. Med. Pharmacol. Sci., 2014, 18, 740-752.

30 X. Zhao, Y. S. Lui, P. W. J. Toh and S. C. J. Loo, Materials (Basel), 2014, 7, 7398-7408.

31 X. Liu, J. M. Holzwarth and P. X. Ma, Macromol. Biosci., 2012, 12, 911-919. 
32 J. Zhang, J. N. Li, G. L. Jia, Y. K. Jiang, Q. Y. Liu, X. Y. Yang and S. Pan, RSC Adv., 2017, 7, 56732-56742.

33 S. Jo, S. M. Kang, S. A. Park, W. D. Kim, J. Kwak and H. Lee, Macromol. Biosci., 2013, 13, 1389-1395.

34 S. Y. Yang, E. S. Kim, G. Jeon, K. Y. Choi and J. K. Kim, Mater. Sci. Eng., C, 2013, 33, 1689-1695.

35 J. Mosnacek, A. Popelka, J. Osicka, J. Filip, M. Ilcikova, J. Kollar, A. B. Yousaf, T. Bertok, J. Tkac and P. Kasak, J. Colloid Interface Sci., 2018, 512, 511-521.

36 A. P. White, A. R. Vaccaro, J. A. Hall, P. G. Whang, B. C. Friel and M. D. McKee, Int. Orthop., 2007, 31, 735-741.

37 W. F. McKay, S. M. Peckham and J. M. Badura, Int. Orthop., 2007, 31, 729-734.

38 A. H. Reddi, Nat. Biotechnol., 1998, 16, 247-252.

39 M. Kretzschmar, J. Doody and J. Massague, Nature, 1997, 389, 618-622.
40 K. Tsuji, A. Bandyopadhyay, B. D. Harfe, K. Cox, S. Kakar, L. Gerstenfeld, T. Einhorn, C. J. Tabin and V. Rosen, Nat. Genet., 2006, 38, 1424-1429.

41 E. Ko, K. Yang, J. Shin and S. W. Cho, Biomacromolecules, 2013, 14, 3202-3213.

42 P. Ducy, M. Starbuck, M. Priemel, J. H. Shen, G. Pinero, V. Geoffroy, M. Amling and G. Karsenty, Genes Dev., 1999, 13, 1025-1036.

43 V. Viereck, H. Siggelkow, S. Tauber, D. Raddatz, N. Schutze and M. Hufner, J. Cell. Biochem., 2002, 86, 348-356.

44 J. B. Lian and G. S. Stein, The Journal of oral implantology, 1993, 19, 95-105; discussion 136-107.

45 D. E. Tolman, Int. J. Oral Maxillofac Implants, 1995, 10, 275294. 